\title{
OPEN Meta-analysis identifies the effect of dietary multi-enzyme supplementation on gut health of pigs
}

\author{
Sivasubramanian Ramani ${ }^{1}{ }^{1}$, Neeraja Recharla ${ }^{1}{ }^{1}$, Okhwa Hwang ${ }^{(2}$, Jinyoung Jeong ${ }^{(22}$ \& \\ Sungkwon Park $\mathbb{1}^{1 凶}$
}

Gut health though is not well defined the role of gastrointestinal tract is vital if an animal must perform well. Apart from digestion, secretion, and absorption gut is harbored with consortium of microbiota which plays a key role in one's health. Enzymes, one of the alternatives for antibiotics with beneficial effects on digestion and consistency of food and its effect on gut health. The effect of enzyme supplementation on gut health is not well established and the objective of this meta-analysis is to investigate if the enzyme supplement has influence on gut. This meta-analysis includes 1221 experiments which has single enzyme studies and or studies with multiple enzyme complexes but not challenged. The ratio of Lactobacillus and $E$. coli is related to ADFI which showed comparatively lower negative correlation coefficient, with -0.052 and -0.035 , respectively, whose $I^{2}$ values are below $25 \%$, showing that these studies show a significantly lower level of heterogeneity. Correlation between villus height, crypt depth, their ratio and fatty acid is also assessed, and it showed that when the animal is supplemented with two enzyme complexes resulted in positive gut health rather than the single or more than two enzymes.

The gastrointestinal tract (GIT) is the interface at which digestion, secretion, and absorption occurs. In, pigs, the digestive system is monogastric ${ }^{1}$. The pig's primitive digestive system is formed when it is at twelve days of gestation period from the entoderm, however, the maturity of the GIT develops during the pig's perinatal life. Most of the digestive capabilities are observed during the perinatal stage ${ }^{2-6}$. Hitherto, there is no known definition for the health of the gut, or gut health. According to the World Health Organization, gut health is broadly defined as follows: (1) effective digestibility and absorption of food, (2) absence of gut illness, (3) normal and stable gut microbiota, (4) good immune status, and (5) a status of well-being ${ }^{7}$.

The focus of global livestock industries is the use of economical, efficient, and sustainable production methods without compromising the quality or quantity of the products, as this determines the potential of obtaining good economic returns. Globally, swine is the most highly consumed meat and also serves as good animal model. Despite many milestones having been achieved in research, there is still room to improve the efficiency of the production of swine $e^{8,9}$. An efficient production using swine is obtained by maintaining the swine from the piglet stage to the slaughter stage with good health and good feed concomitantly without disturbing the animal's welfare or environment ${ }^{10}$. During this process, pigs consume several types of feed and are subjected to various feeding strategies. Moreover, for economic reasons, many alternative feeds have been used that are difficult for the digestion (example, non-digestible fiber rich feed) in a monogastric animal. Furthermore, the use of antibiotics in animal feed as a growth promotor should be restricted due to the possibility of arising antibiotic resistance and environmental issues.

The use of enzymes in feed supplement for promoting gut health in pigs is expected to increase as an alternative to antibiotics and a solution to economic concerns. Enzymes can also be used very effectively as an alternative to probiotics or essential oils ${ }^{11,12}$. Due to this increasing interest, this study is conducted to meet the needs of long-term. Yet, no-good studies are made to investigate the efficacy of enzymes in feed supplement in terms of the gut health of pigs or any livestock animal.

${ }^{1}$ Department of Food Science and Biotechnology, Sejong University, 209 Neungdong-ro, Seoul 05006, Korea. ${ }^{2}$ National Institute of Animal Science, RDA, Jeonju 55365, Jeollabukdo, Korea. ${ }^{凶}$ email: sungkwonpark@ sejong.ac.kr 


\begin{tabular}{|l|l|l|l|l|l|}
\hline Feed type & Complex & Single & Three & Two & Grand total \\
\hline Ad libitum & 380 & 311 & 279 & 68 & 1038 \\
\hline Controlled & & 12 & & 145 & 157 \\
\hline Restricted feed & & 26 & & & 26 \\
\hline Grand total & 380 & 349 & 279 & 213 & 1221 \\
\hline
\end{tabular}

Table 1. Enzyme supplement category based on feeding method.

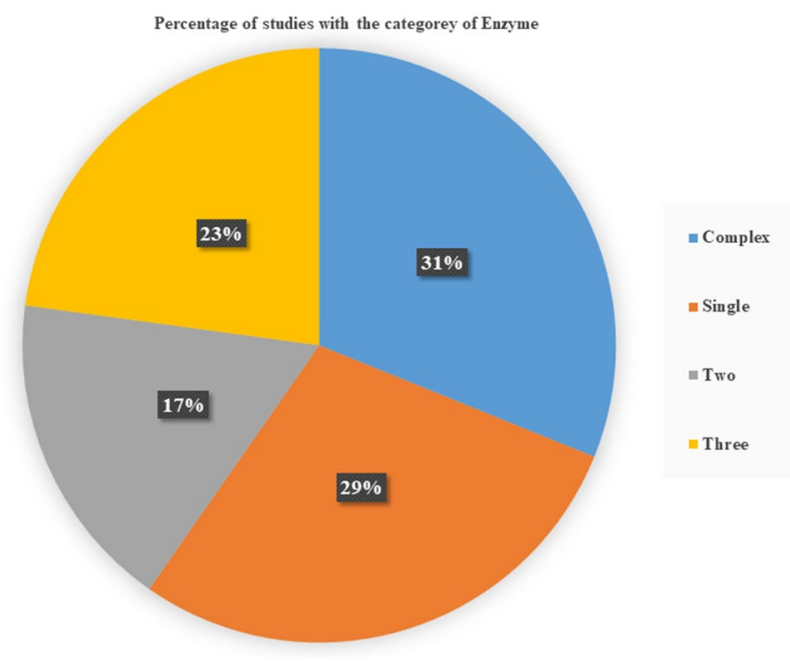

Figure 1. Pie-chart showing the total percentage of the studies by each enzyme supplement category.

Meta-analysis uses a statistical technique in a systematic review to derive and integrate the results from all the included studies ${ }^{13,14}$. The effect size indices help to perform the quantification of all the collected study results using the same metrics. The assessment of heterogeneity is quantified by Cochran $Q$ statistic, $I^{2}, H^{2}$, and ${ }^{15} \tau^{2}$. Density plotting is a good approach for visualizing data in order to confirm whether the data is normally distributed or whether there is any skew or kurtosis.

\section{Results and discussion}

The titles and abstracts of 112 articles are screened before selecting 17 articles (Supplementary 1) for consideration in this study. Overall, 1221 experimental treatments were considered for this study after an elaborate search and duplicates were excluded. The feed system categories are ad libitum, controlled, and restricted feed which are used in 1038, 157, and 26 treatments, respectively. Most of the animal studies involved ad libitum feeding systems. The treatments selected here has at least one type of enzyme supplements, ranging from single enzyme supplements to studies with multiple enzyme complexes. There are 349 treatments in which one type of enzyme is used, 213 treatments in which two types of enzyme were supplemented, 279 treatments in which three types of enzymes were supplemented, and 380 treatments in which more than three types of enzyme were supplemented, categorized as multi-enzyme complex. Table 1 shows the characteristics of the feeding methods with the type of enzyme supplement. The total percentage of the studies for each enzyme category is shown in Fig. 1, as follows: multi-enzyme complex studies made up $31 \%$ of the database, single enzyme supplementation $29 \%$, two-enzyme supplementation $17 \%$, and three-enzyme supplementation $23 \%$.

The selected articles are reported with the different enzyme supplementation which are listed in the Table 2. The supplemented enzymes are categorized into carbohydrase, protease, phytase and lipase. Though many studies included single type of enzyme which are carbohydrase, protease and lipase the individual enzymes are little varied based on the choice of feed, herein carbohydrase are $a$-Galactosidase and xylanase supplemented as single enzymes, and xylanase was always added in combination with other carbohydrase is $\beta$-Glucanase in case of single type of enzyme supplement. Apart from this, some treatments included protease and lipase as individual enzyme supplement. In combination supplemented articles with carbohydrase and protease, xylanase is present in most of the treatments. In case of two types of enzyme supplements, it is 3 different enzymes are feed where 2 enzymes are carbohydrase which are xylanase and amylase. The other type added is protease which is extracted from bacterial source. Phytase is feed in two or three enzyme type combination only.

Most of the experiments are carried using commercial enzymes, accounting for $66.83 \%$ of the treatments, followed by enzymes of bacterial and fungal origin, with $23.58 \%$. These two types of enzymes account for $90.41 \%$ of the database created. On the other hand, treatments where the enzymes of bacterial and fungal origin are 


\begin{tabular}{|c|c|c|c|c|c|}
\hline S. no & Study & Total enzymes & Total enzyme types & Enzyme type & Enzymes \\
\hline 1 & Pan, B. et al., & 1 & 1 & Carbohydrase & a-Galactosidase \\
\hline 2 & Lan, R. et al., & 1 & 1 & Carbohydrase & Xylanase \\
\hline 3 & Passos, A. A. et al., & 1 & 1 & Carbohydrase & Xylanase \\
\hline 4 & Zuo, J. et al., & 1 & 1 & Protease & Protease \\
\hline 5 & Tactacan, G. B. et al., & 1 & 1 & Protease & Protease \\
\hline 6 & Zhang, S. et al., & 1 & 1 & Lipase & Lipase \\
\hline 7 & Owusu-Asiedu, A. et al., & 2 & 1 & Carbohydrase & Xylanase and $\beta$-Glucanase \\
\hline 8 & O'Connell, J. M. et al., & 2 & 1 & Carbohydrase & Xylanase and $\beta$-Glucanase \\
\hline 9 & Jiang, X. R. et al., & 2 & 1 & Carbohydrase & Xylanase and $\beta$-Glucanase \\
\hline 10 & Chen, Q. et al., & 2 & 1 & Carbohydrase & Bacterial Xylanase and Fungal Xylanase \\
\hline 11 & Li, Y. et al., & 3 & 2 & Carbohydrase and Protease & Amylase, Protease and Xylanase \\
\hline 12 & Yi, J. Q. et al., & 3 & 2 & Carbohydrase and Protease & Amylase, Protease and Xylanase \\
\hline 13 & Zhang, G. G. et al., & 3 & 2 & Carbohydrase and Protease & Amylase, Protease and Xylanase \\
\hline 14 & Jo, J. K. et al., & 3 & 2 & Carbohydrase and Protease & Amylase, Mannanase, Protease \\
\hline 15 & Kim, J. et al., & 5 & 3 & Carbohydrase, Protease and Phytase & Amylase, Protease, Mannanase, Xylanase, And Phytase \\
\hline 16 & Kiarie, E. et al., & 6 & 1 & Carbohydrase & $\begin{array}{l}\text { Pectinase, Cellulase, Mannanase, Xylanase, } \beta \text {-Glucanase, } \\
\text { Galactanase }\end{array}$ \\
\hline 17 & Wang, Y. et al., & 6 & 2 & Carbohydrase and Phytase & $\begin{array}{l}\text { Xylanase, } \beta \text {-Glucanase, Cellulase, Pectinase, Amylase and } \\
\text { Phytase }\end{array}$ \\
\hline
\end{tabular}

Table 2. Types of enzymes as feed supplement utilized in the meta-analysis. Reference in the table are in the supplementary document.

individually supplemented accounted for with 5.24\% and 5.48\%, respectively, along with other types of feed administered to the pigs in the different studies, as presented in Table 3.

Effects of enzymes in gut health. An in-depth meta-analysis of digestion, a gut health response variable processed with subclasses average daily feed intake (ADFI), average daily gain (ADG), crude protein (CP), gross energy (GE), gain to feed ratio (G:F) and the Lactobacillus : E. coli (La:Ec) ratio, is conducted. In the case of ADFI, the estimates showed a significantly lower heterogeneity with a total heterogeneity and a standard error (SE) of 0.0055 and 0.0043 , respectively, whose total heterogeneity by total variability, $I^{2}$, is $18.81 \%$. The heterogeneity of $Q$-statistic is 69.0841 , with a p-value of 0.4068 (Table 4 ). The model estimate is -0.0525 with a standard error (SE) of 0.0242 , whose $\mathrm{z}$-score is -2.1726 and a $95 \%$ class interval (CI) upper bound and lower bound of -0.0051 and -0.0998 , respectively. The analysis shows that the studies are not different and hence the results are valid. Overall, the enzyme supplement in the feed, irrelevant on the type and number of enzymes, showed a negative correlation (Table 5). On the other hand, the ADFI and La:Ec subclasses had a lower negative correlation coefficient, with -0.052 and -0.035 , respectively, and a 95\% CI upper bound of -0.005 and 0.011 , respectively, with a lower bound of -0.099 and -0.08 , whose $I^{2}$ values are below $25 \%$, indicating that these studies show a very significant lower level of heterogeneity. Moreover, it is evident that the ratio of Lactobacillus and E. coli is related to the ADFI. This heterogeneity is dependent on animal age, gender, feed, and other conditions. These results are consistent with other available experimental studies displaying that pigs when fed with enzyme supplemented diet has presented with better gut health even when the pigs are challenged with other pathogens ${ }^{16}$. Hence, the outcomes coherent and indicate that feed intake with specific enzymes plays a critical reduction in pathogens (ex. E.coli) or leaky gut, in other words, this can maintain the gut flora with beneficial microbial abundance (ex., Lactobacillus sp.) with the static effects towards pathogenic bacteria which is a protective mechanism for maintenance or enhancement of gut health.

Assessment of publication bias. The publication bias is assessed using funnel plots and further regression tests for funnel plot asymmetry is done in every case (Fig. 2a-g). Using Egger's mixed effects meta regression model, the publication bias is predicted based on the standard error and rank correlation test for asymmetry, using Kendell's Tau statistics for ADFI, ADG, CP, GE, G:F, and La:Ec (Table 6). Egger's test for fatty acids (FA) and La:Ec showed no significance, which indicates that there is no evidence for publication bias in this case. However, although the ADFI and G:F results are non-significant, they are comparatively lower than the former ones. Although, there is no consensus for the beneficiary effects of ADFI, ADG, GE, CP, or G:F in feed supplemented with enzymes, in the case of La:Ec and FA, the effects of enzyme supplementation are observed in some studies ${ }^{17-19}$.

Growth relation with ADFI. The ameliorating effect of enzyme supplementation on feed appears to be based on the substrate which is added. In enzyme supplementation strategy, as the addition of enzymes is assumed to increase animal performance, in this study we carried out variance-covariance analysis to decipher the enzyme supplement efficacy in animal gut health. Polynomial regression analysis is performed using the ADFI with ADG values. Initially, the data distribution is visualized using density plots (Fig. 3a-c). To fit the 


\begin{tabular}{|c|c|c|c|c|}
\hline Count of feed type & Ad libitum & Controlled & Restricted feed & Grand total \\
\hline Complex & 380 & & & 380 \\
\hline Bacteria and Fungi & 148 & & & 148 \\
\hline Corn and SBM & 148 & & & 148 \\
\hline Commercial & 232 & & & 232 \\
\hline Corn and SBM & 57 & & & 57 \\
\hline Corn and SBM with EO & 57 & & & 57 \\
\hline Flaxseed and carbohydrase & 59 & & & 59 \\
\hline No flaxseed and carbohydrase & 59 & & & 59 \\
\hline Single & 311 & 12 & 26 & 349 \\
\hline Bacteria & 59 & 5 & & 64 \\
\hline Corn and SBM & & 5 & & 5 \\
\hline Fish-soybean meal & 33 & & & 33 \\
\hline Soybean based diet & 26 & & & 26 \\
\hline Commercial & 252 & & 26 & 278 \\
\hline $2.5 \%$ soybean oil-Corn and SBM & 36 & & & 36 \\
\hline 5\% soybean oil-Corn and SBM & 36 & & & 36 \\
\hline Corn and SBM & 18 & & 26 & 44 \\
\hline Soybean meal & 162 & & & 162 \\
\hline Fungi & & 7 & & 7 \\
\hline Corn and SBM & & 7 & & 7 \\
\hline Three & 279 & & & 279 \\
\hline Bacteria and Fungi & 122 & & & 122 \\
\hline Corn and SBM & 122 & & & 122 \\
\hline Commercial & 157 & & & 157 \\
\hline Corn and SBM & 107 & & & 107 \\
\hline Stachyose-corn and SBM & 50 & & & 50 \\
\hline Two & 68 & 145 & & 213 \\
\hline Bacteria and Fungi & 18 & & & 18 \\
\hline Mixed grain-based diets & 18 & & & 18 \\
\hline Commercial & 50 & 85 & & 135 \\
\hline Barley wheat-based diets & & 45 & & 45 \\
\hline Corn and SBM & 41 & & & 41 \\
\hline EO and Barley wheat-based diets & & 40 & & 40 \\
\hline Wheat and barley & 9 & & & 9 \\
\hline Fungi & & 60 & & 60 \\
\hline Barley based diet & & 36 & & 36 \\
\hline Wheat based diet & & 24 & & 24 \\
\hline Grand total & 1038 & 157 & 26 & 1221 \\
\hline
\end{tabular}

Table 3. Consolidated table with feed type and method, enzyme type and its origin. Flow of information through the stages of the meta-analysis.

\begin{tabular}{|c|c|c|c|c|c|c|c|c|c|c|c|c|c|c|}
\hline & \multicolumn{8}{|c|}{ Test of heterogeneity } & \multicolumn{6}{|c|}{ Model results } \\
\hline & $k$ & $\tau^{2}$ & se & $\tau$ & $I^{2}$ & $H^{2}$ & Q-statistic & $p$-value & Estimate & se & $z$-score & $p$-value & ci.lb & ci.ub \\
\hline ADFI & 68 & 0.0055 & 0.0043 & 0.0739 & 18.81 & 1.23 & 69.0841 & 0.4068 & -0.0525 & 0.0242 & -2.1726 & 0.0298 & -0.0998 & $-0.0051^{*}$ \\
\hline ADG & 67 & 0.0406 & 0.0151 & 0.2015 & 63.56 & 2.74 & 153.471 & $<.0001$ & -0.2214 & 0.0397 & -5.5819 & $<.0001$ & -0.2992 & $-0.1437^{\star * *}$ \\
\hline $\mathrm{CP}$ & 54 & 0.1029 & 0.0376 & 0.3207 & 69.1 & 3.24 & 133.5287 & $<.0001$ & -0.3676 & 0.0621 & -5.9178 & $<.0001$ & -0.4893 & $-0.2458^{* * *}$ \\
\hline GE & 40 & 0.1399 & 0.0548 & 0.374 & 73.94 & 3.84 & 106.5291 & $<.0001$ & -0.5258 & 0.0798 & -6.5926 & $<.0001$ & -0.6821 & $-0.3695^{* * *}$ \\
\hline G:F & 68 & 0.084 & 0.0284 & 0.2899 & 61.31 & 2.58 & 150.7349 & $<.0001$ & -0.1753 & 0.0512 & -3.4271 & 0.0006 & -0.2756 & $-0.0750^{* * *}$ \\
\hline La:Ec & 53 & 0.0046 & 0.0042 & 0.0681 & 18.84 & 1.23 & 40.9558 & 0.8652 & -0.0345 & 0.0231 & -1.4941 & 0.1351 & -0.0798 & 0.0108 \\
\hline
\end{tabular}

Table 4. Random-effects model with the $\tau^{2}$ estimator as restricted maximum-likelihood estimator. Significant

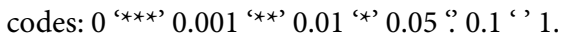




\begin{tabular}{|l|l|l|l|l|l|l|l|l|l|l|l|l|l|l|l|l|l|}
\hline & Correlation & ci.lb & ci.ub & cr.lb & cr.ub & $\boldsymbol{\tau} \mathbf{2}$ & ci.lb & ci.ub & $\boldsymbol{\tau}$ & ci.lb & ci.ub & I2 & ci.lb & ci.ub & H2 & ci.lb & ci.ub \\
\hline ADFI & -0.052 & -0.099 & -0.005 & -0.202 & 0.1 & 0.0055 & 0 & 0.0143 & 0.0739 & 0 & 0.1195 & 18.8122 & 0 & 37.7129 & 1.2317 & 1 & 1.6055 \\
\hline ADG & -0.218 & -0.291 & -0.143 & -0.554 & 0.179 & 0.0406 & 0.0459 & 0.2299 & 0.2015 & 0.2143 & 0.4795 & 63.5586 & 66.3639 & 90.8107 & 2.7441 & 2.973 & 10.8822 \\
\hline CP & -0.352 & -0.454 & -0.241 & -0.765 & 0.266 & 0.1029 & 0.0491 & 0.2407 & 0.3207 & 0.2215 & 0.4906 & 69.0993 & 51.6135 & 83.9576 & 3.2362 & 2.0667 & 6.2335 \\
\hline GE & -0.482 & -0.593 & -0.354 & -0.855 & 0.22 & 0.1399 & 0.0587 & 0.3085 & 0.374 & 0.2423 & 0.5554 & 73.9358 & 54.346 & 86.2176 & 3.8367 & 2.1904 & 7.2557 \\
\hline G:F & -0.174 & -0.269 & -0.075 & -0.636 & 0.381 & 0.084 & 0.0431 & 0.1887 & 0.2899 & 0.2076 & 0.4344 & 61.3083 & 44.8259 & 78.0651 & 2.5845 & 1.8124 & 4.5589 \\
\hline La:Ec & -0.035 & -0.08 & 0.011 & -0.174 & 0.106 & 0.0046 & 0 & 0.0023 & 0.0681 & 0 & 0.0475 & 18.8399 & 0 & 10.1631 & 1.2321 & 1 & 1.1131 \\
\hline
\end{tabular}

Table 5. Summary of correlation for Predicted Fisher's r-to-z scores transformed to correlation coefficients.

model, the $x$ variable is cubed when the $y$ represented ADFI with an intercept $(\alpha)$ of $0.001(\mathrm{p}=0.01)$ and where $\beta_{1}$ is $1.118, \beta_{2}$ is 1.346 , and $\beta_{3}$ is 0.117 (Fig. $4 \mathrm{a}$ ). This fitted model had a residual standard error (RSE) of 0.2383 and 56 degrees of freedom (DOF), a multiple R-squared (RSD) of 0.1965, an adjusted R-squared (Rad) of 0.1534 with a F-statistic of 4.564 ( $\mathrm{p}$-value $=0.006256$ ). Reduction in ADG is ascribed to a reduced ADFI, however in the gut health challenges, growth may be impaired due to an increase in the requirements for the metabolic and digestive processes. Apart from gut health conditions, the G:F ratio also is impaired by other factors like diet composition, age of pigs, other environmental factors also influence. However further studies are needed considering all the factors.

Influence of enzymes on Lactobacillus versus E.coli. Many subjects have demonstrated that under dysbiosis conditions, the abundance of $E$. coli is higher and the amount of beneficiary bacteria belonging to the genera Firmicutes (e.g. Lactobacillus) is reduced. Lactobacillus is highly associated with gut health factors and animal performance ${ }^{20}$. Thus, the ratio of Lactobacillus and E. coli has played an important role in the animal's gut health and overall performance. Here, when Lactobacillus is increased, the abundance of E. coli is found to decrease. The $\alpha$ is -0.057 when $y$ is Lactobacillus, with the $x$ squared to fit the regression model (Fig. $4 \mathrm{~b}$ ). The estimated coefficients are $\beta_{1}$ is -0.575 ( $\mathrm{p}$-value $=0.008$ ) and $\beta_{2}$ is -0.39024 . RSE is 0.309 with 41 DOF. The RSD and Rad are 0.4088 and 0.38 , respectively, with an F-statistic of 14.18 (p-value $=0.00002$ ). Thus, it is very clear that the population of Lactobacillus negatively regulates the pathogenic E. coli population, and the use of enzymes can sometimes enhance the Lactobacillus count in the intestine to improve gut health.

Gut health is associated with microbial abundance and diversity as the presence of certain types of microbes are beneficial, since the metabolites produced by these bacteria assist other beneficial bacteria in the microbial network $\mathrm{k}^{20-23}$. However, the influence of the types or numbers of enzymes on the structure of microbes and fatty acids production in gut has not yet been well established. The changes in substrate availability for microbes is a determining factor in the role of microbes in gut health. In the case of $E$. coli, model fitting did not show any trend or significance regarding fatty acids in the GIT (Fig. 4c). On the other hand, Lactobacillus showed a trend and model fitting (Fig. 4d). The intercepts for E. coli and Lactobacillus are 0.2437 (p-value $=0.000547$ ) and -0.3829 (p-value $=0.000004$ ), respectively, indicating that when $E$. coli is at zero, the fatty acid intercept is positive, meaning that the production of FA is not dependent on E. coli. On the other hand, the Lactobacillus intercept is predicted to be a negative value, indicating that if Lactobacillus is absent, the production of fatty acid is also absent, which could lead to significant problem. The results are consistent with other published articles where the supplementing the animals with enzyme resulting in reduced pathogens even during challenged conditions and in non-challenged conditions ${ }^{16,24}$.

Changes in VH:CD ratio. Digested food is absorbed through brush borders, also known as projected villi, and invaginated crypts, which are microanatomical structures that play a crucial role in gut health. These structures are lined with a mucus layer that provides a mucosal barrier. The height of the projected villus and the depth of the crypts are also indicators of GIT health. Many factors influence the villus height and crypt depth ratio (VH:CD), including the type of feed, viscosity, $\mathrm{pH}$, and pathological conditions, among others. Thus, a balance between the physiological and pathophysiological is essential for an adequate VH:CD ratio. The supplementation feed with enzymes may modulate the VH:CD levels, with certain studies reporting that enzyme supplementation increases viscosity, leading to changes in this ratio which result in a leaky bowl ${ }^{18}$. In the case of VH:CD, the intercept coefficient is -0.33308 with an RSE of 0.1921 and DOF of 37 (Fig. 4e). The RSD is 0.1706 , $\mathrm{Rad}$ is 0.1257 , and the F-statistic is 3.805 ( $\mathrm{p}$-value $=0.03$ ). The correlation between VH and fatty acid is predicted to have an intercept of -0.20688 (p-value $=0.0000007$ ) with an RSE of 0.174 and a DOF of 37 (Fig. 4f). The RSD is 0.01201 , $\mathrm{Rad}$ is -0.04139 , and the F-statistic is 0.225 ( $\mathrm{p}$-value $=0.79$ ).

This study will serve as an important contribution to the body of literature on the impact of enzyme used in animal feed. Here upon compiling the gut microbial structure (Fig. 5), VH, CD, E. coli, Lactobacillus, and fatty acids against the different types of enzyme complex, and using Hedge's $g$ for comparison, it is very clear that the usage of two types of enzymes, based on the substrate provided, is more beneficial than the use of a single enzyme or three enzymes and above (Fig. 6). It is also clear that the choice of enzymes based on the substrate is critical in case of animal health. For instance, phytase with carbohydrase supplement improves animal performance by increasing mineral and nutrient absorption. the use of enzymes by animal weight category also plays a role ${ }^{25}$. In case of single enzyme supplement for example xylanase (carbohydrase), animals below $10 \mathrm{~kg}$ has showed reduced gut health conditions, where the animals showed reduced V:C ratio mediated by a proinflammatory 


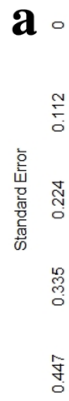

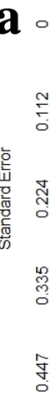
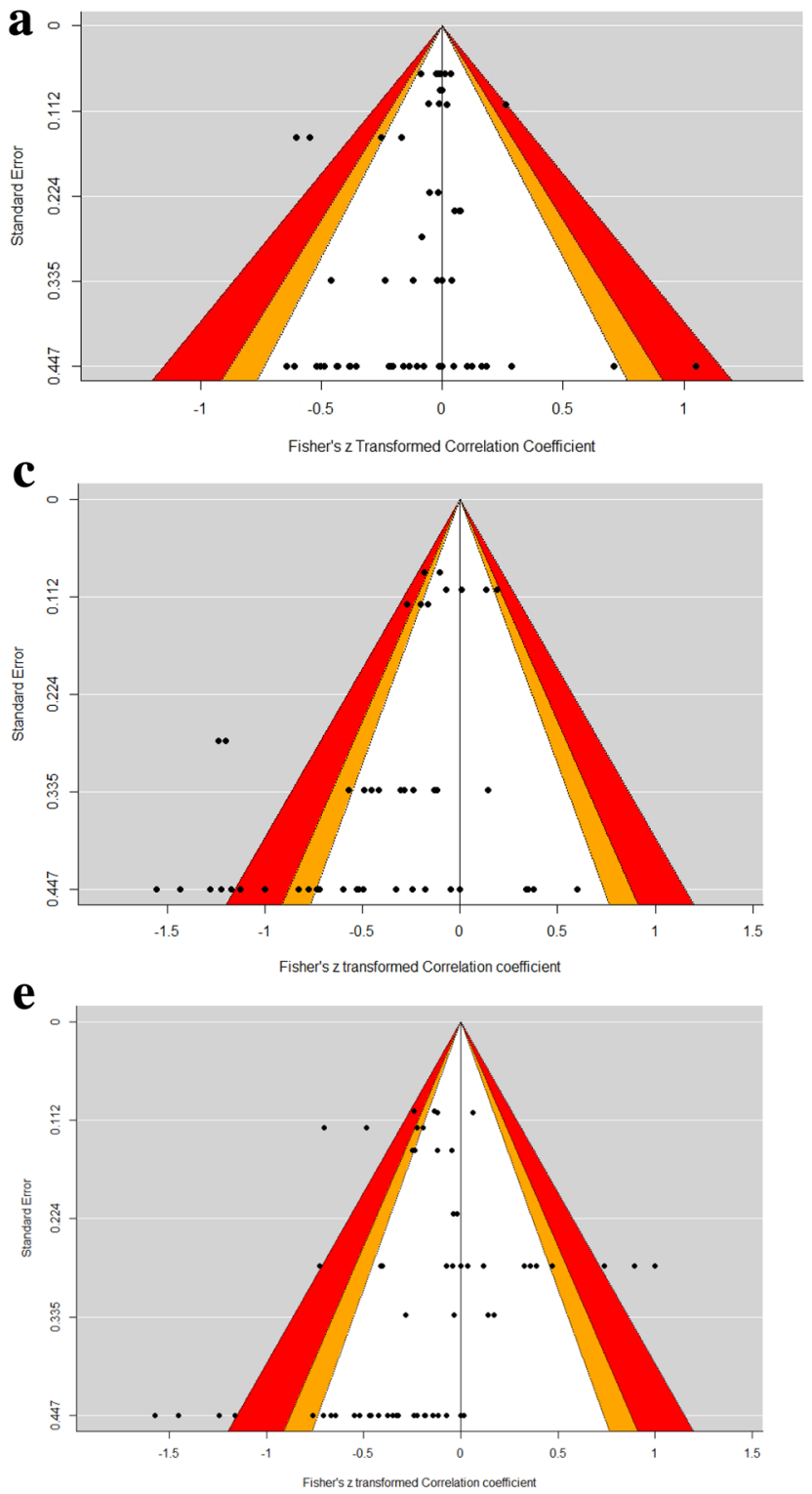

b

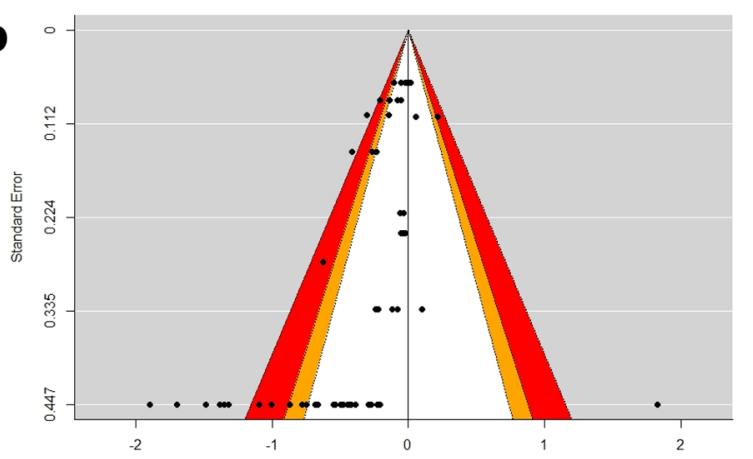

d

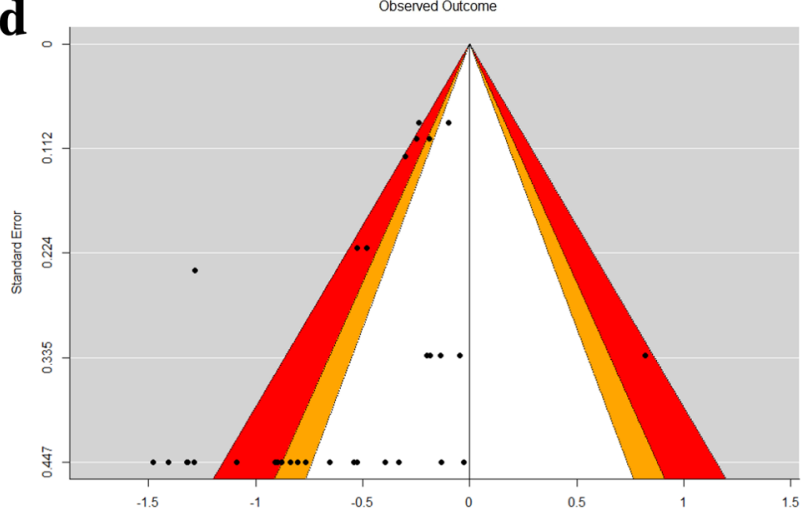

f

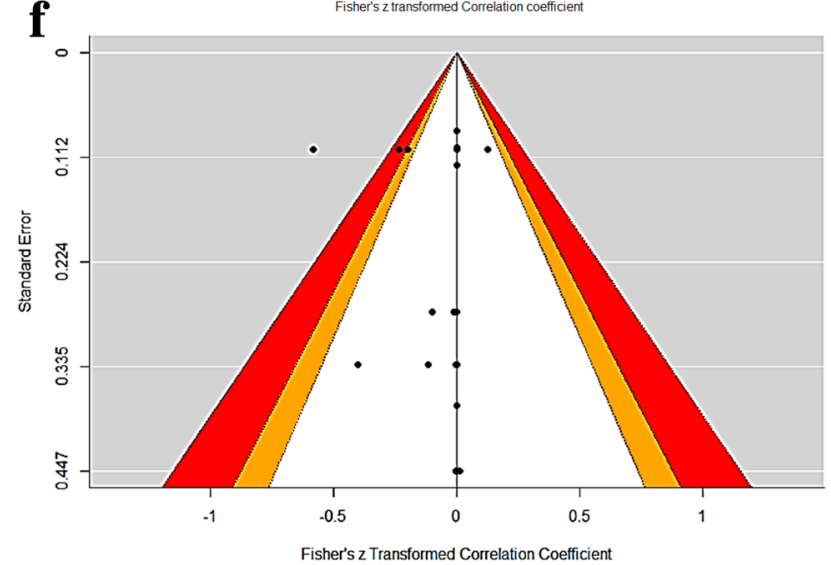

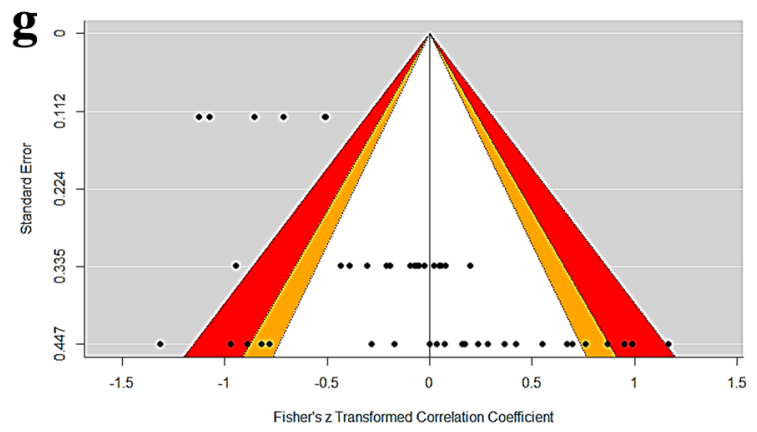

Figure 2. Funnel plots to assess the publication bias. (a) ADFI. (b) ADG. (c) CP. (d) GE. (e) GF. (f) La:Ec. (g) Fatty acids. ADFI: Average daily feed intake; ADG: Average daily gain; LB: Lactobacillus; Ec: Escherichia coli; V/VH: Villus height, C: Crypt depth, CP: Crude protein; GE: Gross energy; GF: Gain to Feed ratio, and La:Ec: Lactobacillus : E. coli La:Ec ratio. 


\begin{tabular}{|l|l|c|l|r|}
\hline \multirow{2}{*}{} & \multicolumn{2}{|l|}{ Eggers regression } & \multicolumn{2}{l|}{ Rank correlation } \\
\cline { 2 - 5 } & $\mathbf{z}$ & $\mathbf{p}$-value & Kendall's $\boldsymbol{\tau}$ & $\mathbf{p}$-value \\
\hline ADFI & -2.0393 & 0.0414 & -0.2204 & 0.0153 \\
\hline ADG & -6.1548 & $<0.0001$ & -0.4452 & $<0.0001$ \\
\hline CP & -4.3254 & $<0.0001$ & -0.2876 & 0.0064 \\
\hline GE & -2.8237 & 0.0047 & -0.4209 & 0.0006 \\
\hline G:F & -1.9186 & 0.055 & -0.2704 & 0.0031 \\
\hline La:Ec & 0.0991 & 0.9211 & -0.6564 & $<0.0001$ \\
\hline FA & 1.2411 & 0.2146 & 0.1009 & 0.3092 \\
\hline
\end{tabular}

Table 6. Tests for publication bias.
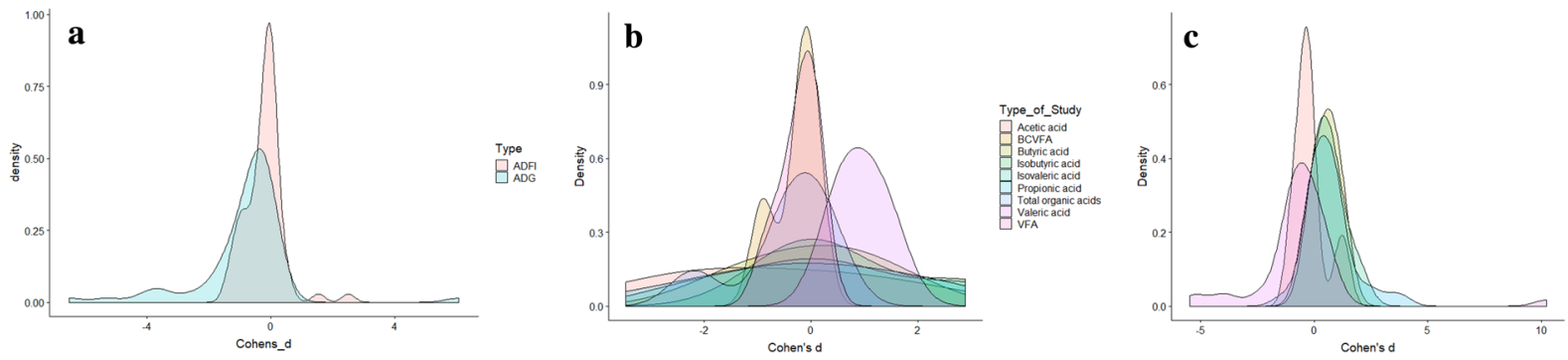

Figure 3. Density plot. (a) Density plot of ADFI and ADG data. (b) Density plot of fatty acids. (c) Density plot of microbes.
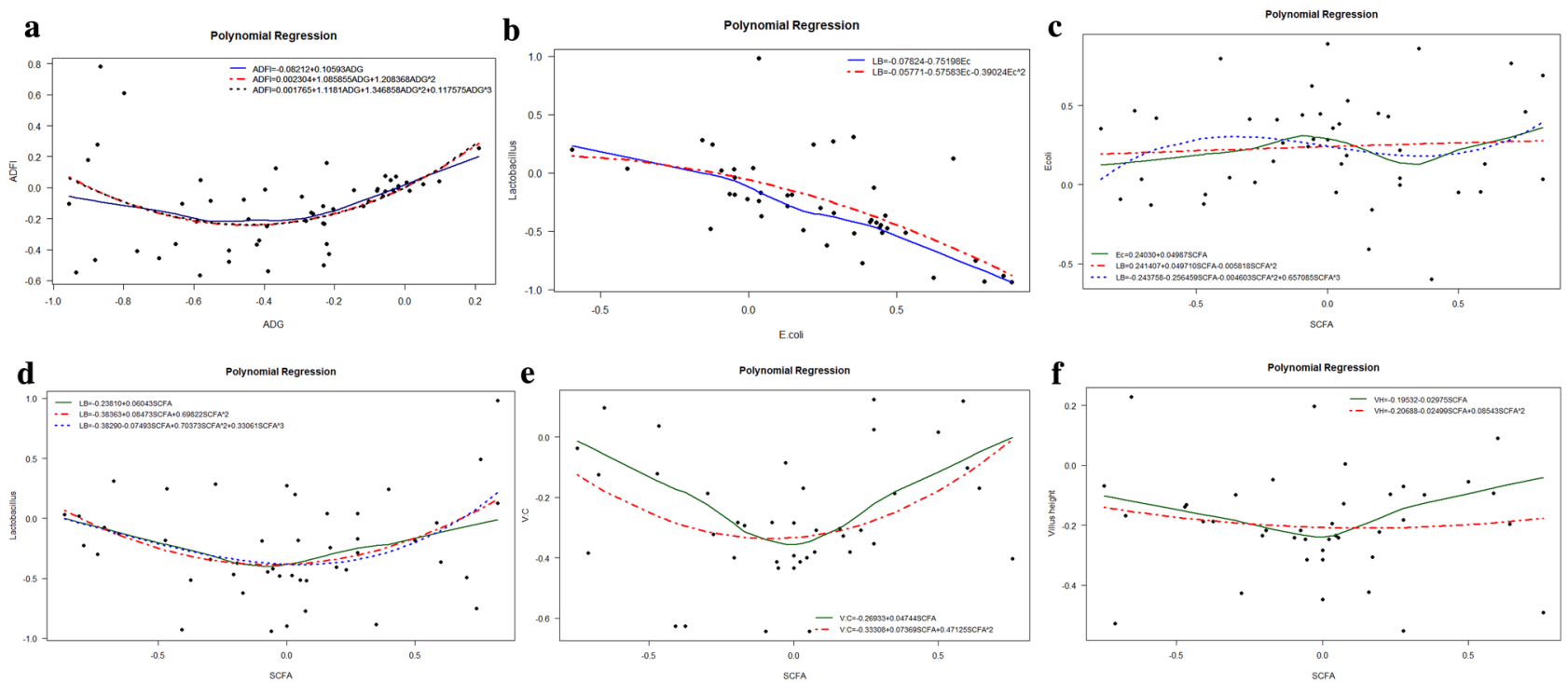

Figure 4. Polynomial regression analysis. (a) Polynomial regression analysis of ADFI versus ADG. (b) Polynomial regression analysis of Lactobacillus versus E.coli (c) Polynomial regression analysis of E.coli versus fatty acids. (d) Polynomial regression analysis of Lactobacillus versu Fatty acids. (e) Polynomial regression analysis of V:C versus fatty acids. (f) Polynomial regression analysis of Villus height versus fatty acids. ADFI: Average daily feed intake; ADG: Average daily gain; LB: Lactobacillus; Ec: Escherichia coli; V/VH: Villus height, C: Crypt depth, FA/SCFA: fatty acids.

cytokine tumor necrosing factor alpha $(\mathrm{TNF} \alpha)^{26}$. TNFa is well known to trigger the inflammatory responses ${ }^{27}$. Whereas, addition of two enzymes like protease and carbohydrase has benefitted the animals by modifications in digestibility and microbial consortium in contrast ${ }^{24}$. Inflammation in gut is mediated through the upregulation of inflammatory cytokines which tends to leaky gut, where the changes in mucosal barrier function, gut immunity, and microorganisms are observed.

To our knowledge there is no meta-analysis reporting the gut health of pigs based on the enzyme supplement. Also, there is a need for more experiments to be proven regarding the activity of enzymes relation with all the gut 


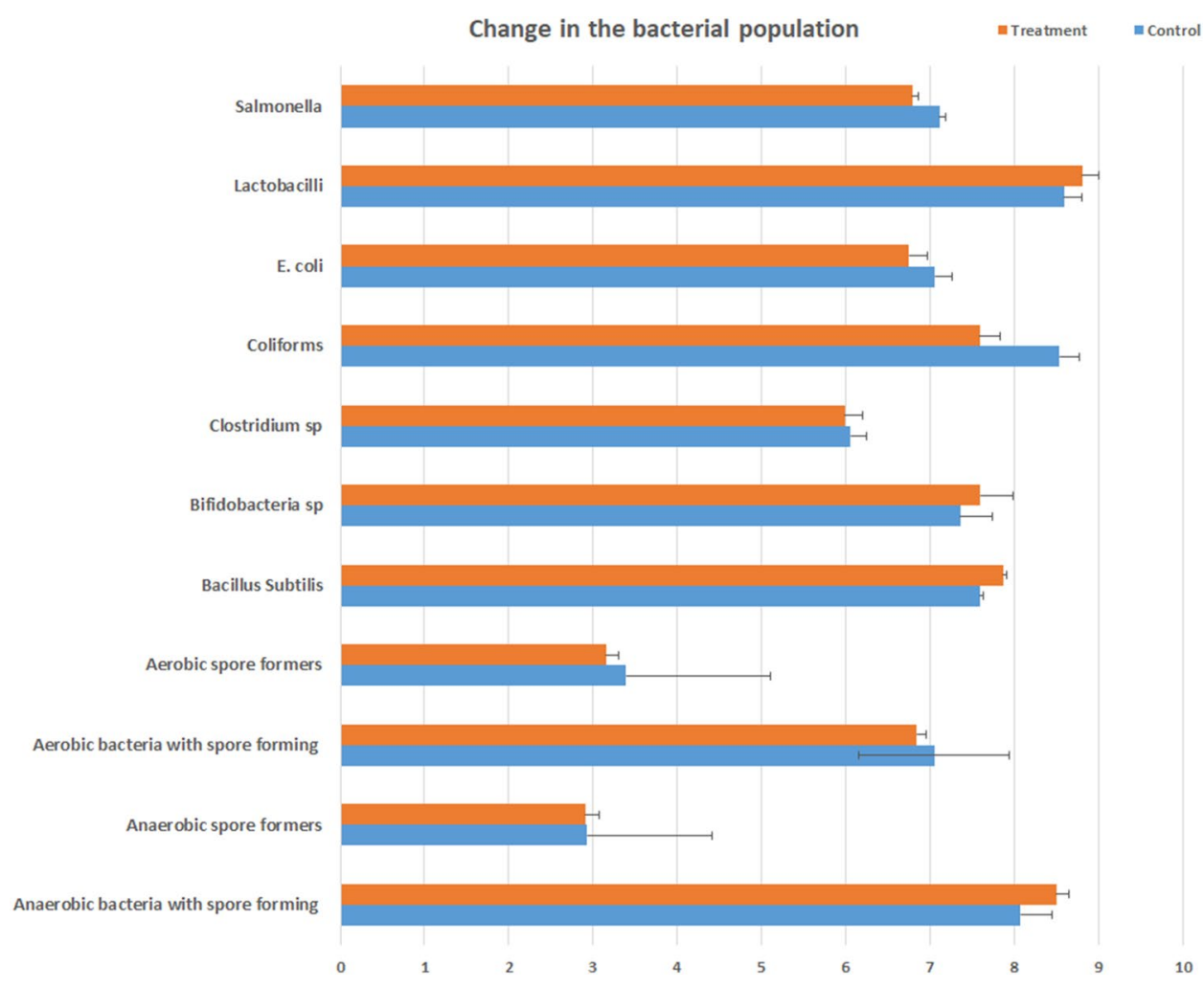

Figure 5. Change in the composition of the gut bacterial population upon enzyme supplement.

\section{Gut health effect changes based on Enzyme suppliment}

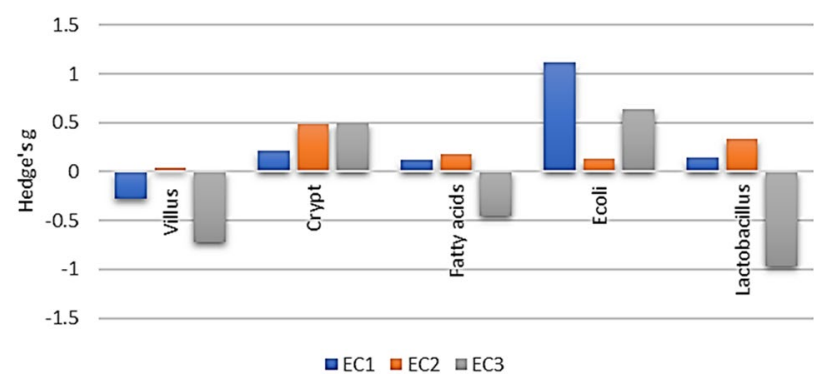

Figure 6. Gut health parameters assessment based on number of enzyme supplement: EC1: single enzyme; EC2: two enzyme complexes; EC3: three or more enzymes.

health attributes. Based on these results, we propose that use of two enzyme supplement in pig feed programs rather than multiple enzyme supplement which may benefit the pig gut health. However further experiments should be conducted to decipher the correlation between enzyme supplement and all the gut health.

\section{Methods}

Search strategy. An intensive computerized literature search is performed to retrieve studies between 2000 and 2018. The search is conducted on PubMed, Web of Science, Google Scholar, and Scopus. The key terms used are "enzymes," "feed additives," "feed supplement," "Swine," "pig," "health," "microorganisms," and "gut health." Upon completing the search, all duplicates are removed, and the articles' titles and abstracts are screened for selection. The full text is then accessed for the knowledge retrieval by two researchers individually. After completing the literature search as per PRISMA guidelines, a flow chart for identification, screening, eligibility, and inclusion is prepared ${ }^{28}$ (Fig. 7). 


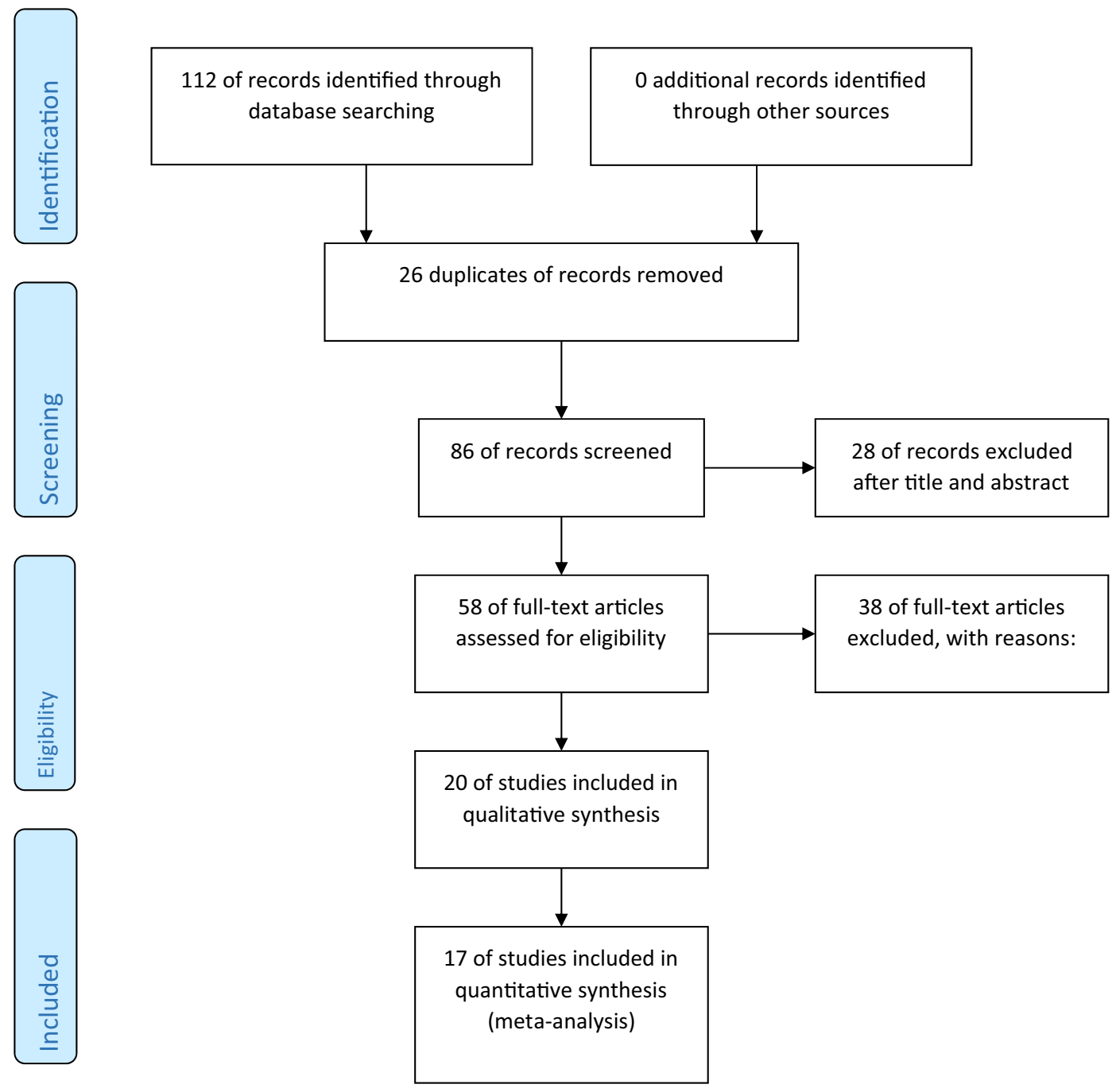

Figure 7. Flow of information through the stages of the meta-analysis.

Literature inclusion and exclusion criteria. Only articles where swine feed is supplemented with enzymes or enzyme complexes and fed to animals that had never been challenged are considered. The article selection criterion is as follows: (1) a weaning period between 17 and 28 days; (2) a commonly used feed, i.e. soybean meal, corn-soybean meal, barley, or wheat, supplemented ad libitum or via a controlled system; (3) feed is supplemented with at least a single type of enzyme or with a multi-enzyme complex; (4) articles reporting animal performance, gut health parameters (such as SCFA), villus and crypt sizes or ratios, and microorganism studies; (5) articles with mean and SEM are only considered to avoid bias. The exclusion criteria are: (1) studies where animals are challenged with pathogens or toxins; (2) feed supplemented with probiotics or prebiotics.

Database characteristics. The database is created using Microsoft Access. A form is created to input the data extracted from each article. Each form represented an experimental study from the selected article which fit as a single row in the database. Initially, the database had a total of 1221 rows and 39 columns. Upon including the data from the articles, the effect sizes are computed and added as separate columns. The calculated effect sizes are Cohen's d, Hedges' g, Fisher's z, Cohen's f, odds ratio, Cox-odds ratio, log odds, Pearson's r, Cox-log odds, effect size eta square, the standard error of the effect size, the variance of effect size, the lower and upper confidence limits, and the weight factor. As a result, the final database had a total of 55 columns after the addition of the calculated effect sizes for meta-analysis. Every experimental study is encoded with a unique number which is generated automatically when a form is generated freshly, which is used as a general code. Each article is given a sequence number. For inter- and intra-studies, another set of sequence number are allocated, such that a unique code is available for each study retrieved from the selected publications. Thus, the rows in the database represented the treatment and the columns represented the exploratory variables or parameters.

Data extraction. All the eligible study data are extracted by data entry using the Microsoft Access form, where the publication year of the article, the title of the article, the author names, the sample size, and the mean 
and SEM are recorded. Moderating variables such as age, weaning period, initial weight, final weight, enzyme type, number of enzymes, enzyme source, enzyme concentration, sample type and time, experiment period, number of animals per pen, genetic background of the animals, feed type, and time are also recorded for analysis. The researchers extracting the data independently cross-verified the data for typographic errors and accuracy.

Data analysis. The unique codified data is classified based on the following five health response variables: absorption and digestion, gut illness, microbiota, immune status, and status of wellbeing ${ }^{7}$. To determine their effects across multiple studies, as well as within individual studies, the models suggested by ${ }^{29,30}$ are utilized. Dependent and independent variables are defined as per the ${ }^{29}$ approach. $\mathrm{R}$ programming version 3.5.1 is used for all data analysis, where the "esc" package is used to calculate the effect size ${ }^{31}$. The calculated effect size is used for the meta-analysis. The packages "metaphor," "robumeta, "meta," "metagear," and/or "mvmeta" are used to conduct the meta-analysis ${ }^{13,32}$. The data is initially explored via graphical analysis to observe the data distribution to check for any heteroscedasticity. Density plots are generated using the Cohen's $\mathrm{d}$ effect size values of the experimental variables.

For conducting a meta-analysis of the effect sizes, the corresponding sample variance are calculated using Cohen's $d$ and Pearson's $r$ ( $r$ ). Here, the $r$ is transformed into Fisher's $z$ score ${ }^{33}$. The correlation coefficient quantifies for both the direction and strength of the linear relationship between the two quantitative variables and is therefore used often as the resulting measure for meta-analyses. Here, the Fisher's r-to-z transformed correlation coefficient is used for the analysis, as this alternative measure is a bias-corrected version of the previous coefficient ${ }^{34}$. The meta-analysis is carried using the random-effects model for measuring between two quantitative variables. Sequentially, the classified data is explored using graphical analysis with Cohen's d, effect size, Fisher's transformed r-to-z, the estimated sample variance, and Hedge's g. Heterogeneity is estimated using the restricted maximum-likelihood estimator method with random-effect model fitting ${ }^{35}$. The study weights are estimated as inverse-variance, which is a default setting in the "metafor" package.

A quantified heterogeneity is reported based on the Q-statistic, $I^{2}, H^{2}$, and $\tau^{2}$ data. A Q-statistic is reported with a DOF with a p-value of the test, which is null hypothesis significance test, which tells us the overall heterogeneity between the studies, but cannot tell us the extent of true heterogeneity ${ }^{36}$. To overcome the limitations of the $Q$-statistic, $\tau^{2}$ is reported to estimate the total amount of true heterogeneity. In the case of $\tau^{2}$, it is only dependent on a specific type of effect size estimates, as such, it cannot be used to compare different meta-analyses with different effect sizes. However, the $I^{2}$ index can be obtained with a different number of studies and with several types of effect size metrics, which are comparable. $I^{2}$ indices of $25 \%, 50 \%$, and $75 \%$ are interpreted as low, moderate, and high, respectively. As shown in previous studies, the between-study variance, $\tau^{2}$, and the $I^{2}$ are directly related ${ }^{15,37}$. The summary of effect size is derived from the estimated model coefficient using the standard error, $\mathrm{z}$-score, $\mathrm{p}$-value, and upper and lower bounds of the confidence interval (CI). For every subgroup metaanalysis is conducted, the results are obtained by further transformation of Fisher's r-to-z scores into correlation coefficients estimates along using the upper and lower bound 95\% CI to interpret the results.

Data visualization. Although a possible heterogeneity is suggested, the data did not point out the specific studies which are influencing this heterogeneity. Thus, Bajaut graphical plotting is carried as a diagnostic plot in an attempt to sort the heterogeneity data. The data used for plotting is the squared Pearson's residuals of the studies and the influencers, i.e. the standardized squared difference between the fitted value for each study. The data points in the plot that identify the influencing data are the study ID numbers. The most influential studies are observed in the top right quadrant of the $\operatorname{plot}^{38}$. Another set of diagnostic plots is also generated and visualized for the potential influencers and outliers using influence plots. Upon computing, we produced a tabular data displaying the difference in the fits, covariance ratios, Cook's distances, and the diagonal elements of the hat matrices. Any influencing data is marked with an asterisk and the influencers are visualized by different colors in the $\operatorname{plot}^{39,40}$.

The point estimates of the study, with the CI and the summary of effect sizes, is plotted on a forest plot to visualize the meta-analysis of the gut health subgroups, or response variables (forest plots are not shown rather the data is presented in table form). The edges of the polygon represent the $95 \%$ confidence limit. The publication bias is evaluated based the funnel plots, the Egger's regression test, and the rank correlation test ${ }^{41}$. Moderator analysis is carried out to estimate the heterogeneity using meta-regression models.

Variance-covariance analysis are performed to determine the effect of enzyme supplementation on the gut health response variables within individual studies and between different studies in which residual analysis is initially conducted. The residual analysis is further graphically verified and observed for normal distribution. The wellbeing of the animals is correlated with performance, hence, the correlation between the coefficients of average daily feed intake is initially regressed against the average daily weight gain to evaluate the biological relevance ${ }^{29}$. Furthermore, to infer the correlation between other gut health factors, polynomial regression model analysis is conducted, as discussed earlier. Factors included comparing Lactobacillus to Escherichia coli, whose populations indicate the status of the health and dysbiosis condition, since gut health can also be characterized by the villus height $(\mathrm{VH})$, crypt depth (CD), concentration of short chain fatty acids (SCFA), and VH:CD ratios. These parameters are mechanistically inter-related in the support of gut health, hence, we verified their relationship and their associated regulatory mechanisms in the presence of enzyme supplements. The formula for the polynomial regression fit is provided along with the representative plots ${ }^{30}$.

Received: 23 March 2020; Accepted: 29 January 2021

Published online: 31 March 2021 


\section{References}

1. Gonzalez, L. M., Williamson, I., Piedrahita, J. A., Blikslager, A. T. \& Magness, S. T. Cell lineage identification and stem cell culture in a porcine model for the study of intestinal epithelial regeneration. PLoS ONE 8, e66465 (2013).

2. Xu, R., Wang, F. \& Zhang, S. Postnatal adaptation of the gastrointestinal tract in neonatal pigs: a possible role of milk-borne growth factors. Livest. Prod. Sci. 66, 95-107 (2000).

3. Buddington, R. K., Sangild, P. T., Hance, B., Huang, E. Y. \& Black, D. D. Prenatal gastrointestinal development in the pig and responses after preterm birth. J. Anim. Sci. 90, 290-298 (2012).

4. Kelly, D., Smyth, J. A. \& Mccracken, K. J. Digestive development of the early-weaned pig. Br. J. Nutr. 65, 181 (1991).

5. Manners, M. J. The development of digestive function in the pig. Proc. Nutr. Soc. 35, 49-55 (1976).

6. Pond, W. G., Maner, J. H. \& Harris, D. L. Pork Production Systems. (Springer US, 1991). https://doi.org/10.1007/978-1-4615-3932-2.

7. Bischoff, S. C. 'Gut health': a new objective in medicine?. BMC Med. 9, 24 (2011).

8. McGlone, J. The future of pork production in the world: towards sustainable, welfare-positive systems. Animals 3, 401-415 (2013).

9. Merchant, H. A. et al. Assessment of gastrointestinal $\mathrm{pH}$, fluid and lymphoid tissue in the guinea pig, rabbit and pig, and implications for their use in drug development. Eur. J. Pharm. Sci. 42, 3-10 (2011).

10. Gasa, J. Feeding strategies in pig production : Sows and their piglets. Anim. Feed Sci. Technol. 233, 34-52 (2017).

11. Wang, Y. et al. Effect of diet complexity, multi-enzyme complexes, essential oils, and benzoic acid on weanling pigs. Livest. Sci. 209, 32-38 (2018).

12. Jiang, X. R. et al. Effects of a blend of essential oils and an enzyme combination on nutrient digestibility, ileum histology and expression of inflammatory mediators in weaned piglets. Animal 9, 417-426 (2015).

13. Quintana, D. S. From pre-registration to publication: a non-technical primer for conducting a meta-analysis to synthesize correlational data. Front. Psychol. 6, 1-9 (2015).

14. Moher, D. et al. Preferred reporting items for systematic reviews and meta-analyses: the PRISMA statement. J. Chin. Integr. Med. 7, 889-896 (2009).

15. Huedo-Medina, T. B., Sánchez-Meca, J., Marín-Martínez, F. \& Botella, J. Assessing heterogeneity in meta-analysis: Q statistic or I 2 Index?. Psychol. Methods 11, 193-206 (2006).

16. Li, Q. et al. Dietary soluble and insoluble fiber with or without enzymes altered the intestinal microbiota in weaned pigs challenged with enterotoxigenic E. coli F18. Front. Microbiol. 11, 1110 (2020).

17. Murphy, P. et al. Effects of cereal $\beta$-glucans and enzyme inclusion on the porcine gastrointestinal tract microbiota. Anaerobe 18, 557-565 (2012).

18. Yi, J. Q. et al. The effects of enzyme complex on performance, intestinal health and nutrient digestibility of weaned pigs. Asian-Aust. J. Anim. Sci. 26, 1181-1188 (2013).

19. Gresse, R. et al. Gut microbiota dysbiosis in postweaning piglets: understanding the keys to health. Trends Microbiol. 25, 851-873 (2017).

20. Ramayo-Caldas, Y. et al. Phylogenetic network analysis applied to pig gut microbiota identifies an ecosystem structure linked with growth traits. ISME J. 10, 2973-2977 (2016).

21. Derrien, M., Belzer, C. \& de Vos, W. M. Akkermansia muciniphila and its role in regulating host functions. Microb. Pathog. 106, 171-181 (2017).

22. Sun, J. \& Dudeja, P. K. Mechanisms Underlying Host-Microbiome Interactions in Pathophysiology of Human Diseases. Mechanisms Underlying Host-Microbiome Interactions in Pathophysiology of Human Diseases (Springer US, 2018). https://doi.org/10.1007/ 978-1-4939-7534-1.

23. Kiarie, E., Romero, L. F. \& Nyachoti, C. M. The role of added feed enzymes in promoting gut health in swine and poultry. Nutr. Res. Rev. 26, 71-88 (2013).

24. Recharla, N. et al. Dietary multi-enzyme complex improves in vitro nutrient digestibility and hind gut microbial fermentation of pigs. PLoS ONE 14, e0217459 (2019).

25. Olukosi, O. A., Sands, J. S. \& Adeola, O. Supplementation of carbohydrases or phytase individually or in combination to diets for weanling and growing-finishing pigs1. J. Anim. Sci. 85, 1702-1711 (2007).

26. Chen, H., Zhang, S. \& Kim, S. W. Effects of supplemental xylanase on health of the small intestine in nursery pigs fed diets with corn distillers' dried grains with solubles. J. Anim. Sci. 98, 1-31 (2020).

27. Van Hauwermeiren, F. et al. TNFR1-induced lethal inflammation is mediated by goblet and Paneth cell dysfunction. Mucosal Immunol. 8, 828-840 (2015).

28. Moher, D., Liberati, A., Tetzlaff, J., Altman, D. G. \& PRISMA Group. Preferred reporting items for systematic reviews and metaanalyses: the PRISMA statement. Ann. Intern. Med. 151, 264-9, W64 (2009).

29. Sauvant, D., Schmidely, P., Daudin, J. J. \& St-Pierre, N. R. Meta-analyses of experimental data in animal nutrition. Animal 2, 1203-1214 (2008).

30. St-Pierre, N. R. Invited review: integrating quantitative findings from multiple studies using mixed model methodology. J. Dairy Sci. 84, 741-755 (2001).

31. Lüdecke, D. esc: Effect Size Computation for Meta Analysis. R package Version 0.4.1 (2018). 10.5281/zenodo.1249218.

32. Polanin, J. R., Hennessy, E. A. \& Tanner-Smith, E. E. A review of meta-analysis packages in R. J. Educ. Behav. Stat. 42, 206-242 (2017).

33. Fisher, R. A. On the " probable error " of a coefficient of correlation deduced from a small sample. Metron 1, 3-32 (1921).

34. Cooper, H., Hedges, L. V. \& Valentine, J. C. The Handbook of Research Synthesis and Meta-analysis (Russell Sage Foundation, 2009).

35. Jennrich, R. I., Jennrich, R. I. \& Sampson, P. F. Newton-Raphson and related algorithms for maximum likelihood variance component estimation. Technometrics 18, 11-17 (1976).

36. Cochran, W. G. The combination of estimates from different experiments. Biometrics 10, 101-129 (1954).

37. Higgins, J. P. T. \& Thompson, S. G. Quantifying heterogeneity in a meta-analysis. Stat. Med. 21, 1539-1558 (2002).

38. Baujat, B., Mahé, C., Pignon, J.-P. \& Hill, C. A graphical method for exploring heterogeneity in meta-analyses: application to a meta-analysis of 65 trials. Stat. Med. 21, 2641-2652 (2002).

39. Viechtbauer, W. Conducting meta-analyses in R with the metafor Package. J. Stat. Softw. 36, 1-48 (2015).

40. Viechtbauer, W. 'metafor'-Meta-Analysis Package for R. CRAN (2017).

41. Egger, M., Smith, G. D., Schneider, M. \& Minder, C. Bias in meta-analysis detected by a simple, graphical test. BMJ 315, 629-634 (1997).

\section{Acknowledgements}

This work is carried out with the support of "Cooperative Research Program for Agriculture science \& Technology Development (Project No. PJ015002032020)” Rural Development Administration and Basic Science Research Program through the National Research Foundation of Korea (NRF) funded by the Ministry of Science, ICT \& Future planning (2019R1F1A1059043), South Korea). 


\section{Author contributions}

S.R. and N.R. collected data and performed meta-analysis. O.H. and J.J. did interpretation of data. J.J. made a conception of this manuscript. O.H. and S.P. designed the experiment and O.H., S.R., N.R. and S.P. article writing. All authors reviewed the manuscript, approved the submitted version, and agreed to their own contribution ensuring questions are appropriately investigated, revolved, and the resolution documented in the literature.

\section{Competing interests}

The authors declare no competing interests.

\section{Additional information}

Supplementary Information The online version contains supplementary material available at https://doi.org/ 10.1038/s41598-021-86648-7.

Correspondence and requests for materials should be addressed to S.P.

Reprints and permissions information is available at www.nature.com/reprints.

Publisher's note Springer Nature remains neutral with regard to jurisdictional claims in published maps and institutional affiliations.

(c) (i) Open Access This article is licensed under a Creative Commons Attribution 4.0 International License, which permits use, sharing, adaptation, distribution and reproduction in any medium or format, as long as you give appropriate credit to the original author(s) and the source, provide a link to the Creative Commons licence, and indicate if changes were made. The images or other third party material in this article are included in the article's Creative Commons licence, unless indicated otherwise in a credit line to the material. If material is not included in the article's Creative Commons licence and your intended use is not permitted by statutory regulation or exceeds the permitted use, you will need to obtain permission directly from the copyright holder. To view a copy of this licence, visit http://creativecommons.org/licenses/by/4.0/.

(c) The Author(s) 2021 